\title{
Tetraploidy enhances the cold-resistance in yellow kiwifruit (Actinidia chinensis)
}

Yipei Li

Southwest Forestry University

Xiaozhen Liu

Southwest Forestry University

Zhou Wei

Southwest Forestry University

Zhimin Zhang

Southwest Forestry University

Wen Bian

Southwest Forestry University

Shengxing Li

Southwest Forestry University

Hanyao Zhang ( $\nabla$ zhanghanyao@hotmail.com)

Southwest Forestry University https://orcid.org/0000-0001-7440-4348

Research article

Keywords: Yellow kiwifruit, tetraploid, cold tolerance, transcriptome, RT-qPCR.

Posted Date: January 17th, 2020

DOI: https://doi.org/10.21203/rs.2.21136/v1

License: (c) (i) This work is licensed under a Creative Commons Attribution 4.0 International License. Read Full License 


\section{Abstract}

Yellow kiwifruit (Actinidia chinensis) is highly susceptible to severe weather, such as low temperature and frost, which may affect the production in the coming year. And the cold-resistant mechanism of kiwifruit associated with gene regulation is poorly investigated. To botain cold-resistant germplam, to provide insight into the causes of differences in low temperature tolerance due to ploidy and to better understand cold-adaptive mechanisms in tetraploid kiwifruit, the diploid yellow kiwifruit 'SWFU03' and its tetraploid plantlets were subjected to cold-tolerant screening with L-hydroxyproline (LHyp) and low temperature, the selected ones were then analyzed by transcriptome data and confirmed by RT-qPCR. The results showed that the survival rate of tetraploid plants was $62.22 \%$ when treated with $8 \mathrm{mmol} / \mathrm{L} \mathrm{L-Hyp}$ for 30 days, while all the diploid ones died. After treated with $0{ }^{\circ} \mathrm{C}$ for $12 \mathrm{~h}$, then at room temperature for seven days, the survival rate of tetraploid plantlets was $42.22 \%$, while all diploidy died. Hence, cold tolerance of the tetraploid plantlets was stronger than that of the diploid genotypes. Using these two screening systems, 126 cold-resistant tetraploid tissue culture plantlets were obtained. A total of 1630 differentially expressed genes (DEGs) were identified, of which 619 were up-regulated and 1011 were down-regulated in the low temperature treatment goup. The DEGs enriched in the cold-tolerance related pathways mainly included plant hormone signal transduction, and starch and sucrose metabolism pathway. RT-qPCR analysis confirmed the expression levels of eight up-regulated genes in these pathways in the cold-resistant mutants. In conclusion, this study has identified cold-resistant yellow kiwifruit plantlets and cold-tolerance related genes. Moreover, the dataset got in this study advances our knowledge of the cold-adaptive genes in the regulatory networks and leads to understand the cold tolerance mechanisms in the tetraploid yellow kiwifruit.

\section{Background}

Yellow kiwifruit (Actinidia Chinensis Planch) has sweet and aromatic flesh, and has excellent nutrition and high economic value (Minchin et al., 2003). The fruit of yellow kiwifruit is very popular in markets (Yuan and Wang, 2018). The output of yellow kiwifruit in New Zealand, Italy, Greece, Chile and other countries increased year by year (Minchin et al., 2003; Zhang et al., 2015).

Cold injury is a major problem affecting the growth of yellow kiwifruit (Vallespir et al., 2019). Cold tolerance of yellow kiwifruit is not strong, and so low temperature and frost may result in yield reduction, or plant death (Wu et al., 2009). In addition to improving the cultivation techniques, cultivation of a cold-tolerant kiwifruit variety from breeding can fundamentally solve the problem of freezing injury.

Although the growth of polyploid plants is slow, stress tolerance and the nutrient content are high (Wu et al., 2009, 2011, 2012). Studies on polyploidy induction of fruit trees have been carried out for a long time, and polyploid plants, such as strawberries, kiwifruit, oranges and pears have been cultivated, which have made great contributions to the tolerance of fruittrees and the production of fruittrees (Wu et al., 2011, 2012).

The low-temperature in winter may cause frostbite in kiwifruit, resulting in a decrease of kiwifruit production (Vallespir et al., 2019). Freezing injury and late frost are the main disasters. The low-temperature freezing injury may cause some kiwifruit leaves to wilt, flowers and fruits to fall, delay fruit production, reduce fruit quality, or result in the death of the whole plant. In addition to preventing freezing disasters by cultivation and selecting suitable environments and regions for planting, breeding and cultivation of cold-resistant $A$. chinensis is a fundamental solution (Vallespir et al., 2019). Therefore, it is urgently needed to produce new cold-resistant yellow kiwifruit varieties.

In this study, given the shortcomings of yellow kiwifruit, which is not resistant to low-temperature, to strengthen the cold-tolerance and improve the production, diploid yellow kiwifruit variety 'SWFU03' and its tetraploidy were used as experimental materials. The cold-tolerance experiments were carried out using the tetraploid seedlings induced and identified in our previous research, and the cold-tolerant mutants were screened by using L-hydroxyproline (L-Hyp) and low temperature. To provide insight into the causes of differences in low temperature tolerance and better understand cold-adaptive mechanisms, selected cold-tolerant mutants were subjected to transcriptome analysis and validation by Real time quantitative polymerase chain reaction (RT-qPCR) analysis.

\section{Results}

\section{Screening of cold-tolerant mutants}

In the study, tetraploid aseptic plantlets and diploid aseptic plantlets (as the control) were treated with L-Hyp. After 7 days of $10 \mathrm{mmol} / \mathrm{L}$ L-Hyp treatments, the browning phenomenon was found to appear at the edge of the leaves in the tetraploid and diploid plantlets. After 14 days, some of the leaves in diploid treated with $10 \mathrm{mmol} / \mathrm{L}$ L-Hyp browned, stopped growing or began to fall off. On the other hand, only a few leaves of tetraploid plantlets fell off, and some of the leaf tip edges appeared brown, and the whole plants were still green.

As shown in Table 2 and Table 3, the survival rates of tetraploid plantlets treated with L-hyp were higher than those of diploid plantlets. After 30 days of treatment, the symptoms of freezing injury in tetraploid plantlets were lighter than those of diploidy.

After 30 days of treatment, the survival rates of tetraploid and diploid plntlets were $100 \%$ in MS medium with 0 mmol/L L-Hyp. In MS medium with 2 $\mathrm{mmol} / \mathrm{L}$ L-Hyp, the survival rate of tetraploid plantlets was $100 \%$, and a small number of diploid plantlets died, with a survival rate of $86.87 \%$. In MS medium with $4 \mathrm{mmol} / \mathrm{L}$ L-Hyp, the tetraploid plantlets began to die, with a survival rate of $96.67 \%$, while the survival rate of diploid plantlets was only 33.33\%. In MS medium with $6 \mathrm{mmol} / \mathrm{L}$ L-Hyp, the survival rate of tetraploid plants was $83.33 \%$, but only a small number of diploid plants survived (the 
survival rate was only 20\%). In MS medium with $10 \mathrm{mmol} / \mathrm{L}$ L-Hyp, all diploid plants died,but the survival rate of tetraploid plants was still $53.33 \%$. The survival rate of tetraploid plantlets was significantly higher than that of diploid plantlets (Table 1).

According to the experimental results, it was concluded that the semi-lethal concentration of L-Hyp in tetraploid plants was $8 \mathrm{mmol} / \mathrm{L}$. With the concentration increase of L-Hyp, the survival rate of diploidy decreased significantly, while the survival rate of tetraploidy decreased gradually. And the survival rates of tetraploid plants at high concentrations were significantly higher those of diploid plants. The results showed that the tolerance of tetraploid plants to L-Hyp was stronger than that of diploidy.

After 30 days of treatment, they were transferred to a normal medium, and the changes of fresh weight before and after treatment were recorded (Table 2). Under the concentration of L-Hyp of $0 \mathrm{mmol} / \mathrm{L}$, the increased rate of tetraploidy the fresh weight was $110.79 \%$, and the increase rate of diploidy fresh weight was $114.13 \%$. Under the condition of no L-Hyp stress, diploidy grew faster and increased fresh weight more than tetraploidy. In MS medium with 2 $\mathrm{mmol} / \mathrm{L}$ L-Hyp, the increased rate of fresh weight of tetraploid and diploid was $104.14 \%$ and $93.63 \%$, respectively. The increased rate of fresh weight of tetraploid was higher than that of diploidy, and some diploidy died. Under the stress of $4 \mathrm{mmol} / \mathrm{L}$ L-Hyp, the increase rate of fresh weight of tetraploidy was $101.94 \%$, while that of diploid was $66.24 \%$. Under the stress of $8 \mathrm{mmol} / \mathrm{L} \mathrm{L-Hyp}$, the increase rate of diploid fresh weight was only $10.28 \%$, and that of tetraploidy was $96.83 \%$. Under the stress of $10 \mathrm{mmol} / \mathrm{L}$ L-Hyp, all the diploidy died, no fresh weight increased, and the rate of increase of tetraploidy fresh weight was $75.67 \%$.

The seedling heights of tetraploidy and diploidy were measured before and 30 days after treatment (Table 3 ). The results showed that the height increase rate of diploidy (152.94\%) was higher than that of tetraploidy (140.12\%) without L-Hyp treatment. Under the stress with $4 \mathrm{mmol} / \mathrm{L}$ L-Hyp, the height of diploid plants increased only slightly, and that of tetraploid plants increased, while under the stress of $6 \mathrm{mmol} / \mathrm{L} L-H y p$, the diploid plants basically stopped growing. Under the stress of $8 \mathrm{mmol} / \mathrm{L}$ L-Hyp, the height of diploid plantlets did not increase and the height of tetraploid plants increased slightly. Under the stress with $10 \mathrm{mmol} / \mathrm{L}$ L-Hyp, all the diploid plants died, so there was no seedling height data, and the growth of tetraploid plants basically stopped (Table 4). The results showed that the growth rate of tetraploid plants treated with L-Hyp at different concentrations was higher than that of diploid plants.

\section{Effect of low-temperature treatment on yellow kiwifruit}

As shown in Figure 1, the freezing injury symptoms of tetraploid plantlets were less than that of diploid plantlets. The survival rates of tetraploid and diploid plantlets at $0{ }^{\circ} \mathrm{C}$ for $3 \mathrm{~h}$ were $88.89 \%$ and $17.78 \%$ respectively (Table 5 ). At $0{ }^{\circ} \mathrm{C}$ for $12 \mathrm{~h}$, the survival rate of tetraploid plantlets was $42.22 \%$, and the survival rate of diploidy was only $2.22 \%$. After low-temperature stress, the tetraploid and diploid plantlets were transferred to normal MS medium and placed at room temperature for observation. Seven days later, the control plantlets grew slowly, the leaves wilted, and dead tip and edge of the leaves appeared. However, most of the tetraploid plantlets grew normally, the degree of chilling injury was obviously alleviated, and even no chilling injury occurred. Therefore, the cold tolerance of tetraploid plantlets was higher than that of diploid plantlets.

\section{Results of transcriptome analysis}

\section{Results of sequence data filtering}

The original data of 6 tetraploid samples were shown in Table 6. To improve the accuracy of the analysis results of the transcriptome data, the sequence data were filtered before the data analysis, and the filtered data were shown in Table 6. The sequence data of sample transcriptome was of high quality.

\section{Detection of DEGs}

There were 1630 DEGs between control group and treatment group. By analyzing the DEGs between control group and treatment group, it was found that 619 DEGs were up-regulated and 1011 DEGs were down-regulated in the treatment group compared with the control group (Table 7).

\section{Functional classification and enrichment analysis of DEGs}

According to the results of differential gene detection, the DEGs were classified and enriched in the GO database. GO enrichment analysis could be divided into three main functions including molecular function, cellular component, and biological process. A total of 1025 DEGs were classified into 44 functional categories. There were 10 functional classifications of molecular function, 13 functional classifications of cellular component and 21 functional classifications of biological process (Figure 2).

\section{Functional classification and enrichment analysis of KEGG pathway}

A total of 410 differentially expressed genes were involved in 89 metabolic pathways in control group and treatment group.The top five pathways enriching DEGs were plant hormone signal transduction (including 25 DEGs), phenylpropanoid biosynthesis24 DEGs), plant-pathogen interaction (20 DEGs), carbon metabolism (14 DEGs), and cysteine and methionine metabolism (13 DEGs). Among them, plant hormone signal transduction was the pathway with the largest number of DEGs annotated to this pathway, which belonged to environmental information processing in five branches.

The number of up-regulated DEGs enriched by phenylpropanoid biosynthesis was 16 in the first three pathways of the KEGG metabolic pathway, and 14 DEGs were enriched in plant-pathogen interaction. A total of 11 DEGs were enriched by carbon metabolism. The top three pathways of down-regulation 
were plant hormone signal transduction, enrichment of 19 DEGs, phenylpropanoid biosynthesis, enrichment of eight DEGs, and six DEGs were enriched in carotenoid biosynthesis.

\section{Screening and analysis of cold tolerance related genes}

In this study, the largest number of DEGs was annotated to plant hormone signal transduction pathway. Many cold-tolerance related studies have found that most of the DEGs were enriched in this pathway. Signal transduction pathway plays a key role in the response of plants to low temperature (Janská et al., 2010). Therefore, the DEGs enriched in this pathway might be related to cold tolerance, so the DEGs in this pathway were analyzed in detail. The pathway map (KO04075) was shown in Figure 3.

There were 25 DEGs in the plant hormone signal transduction pathway, of which six were up-regulated. They were CEY00_Acc07445 (disease-related leaf protein), CEY00_Acc03316 (abscisic acid receptor PYL), CEY00_Acc10294 (indole-3-acetic acid-amide synthetase), CEY00_Acc13130 (bZIP transcription factor family protein), CEY00_Acc33627 (TIFY protein) and CEY00_Acc21162 (pathogenesis related protein); 19 DEGs were down-regulated, including CEY00_Acc07128 (auxin-induced protein), CEY00_Acc19743 (CICLE hypothetical protein), CEY00_Acc23775 (containing Cyclin_N domain protein) and CEY00_Acc07415 (indole-3-acetic acid-amide synthase). The genes involved in this pathway were shown in Table 8.

Abscisic acid affects plant cold tolerance and gene expression regulation and can improve plant cold tolerance. Abscisic acid signal transduction was due to the interaction between abscisic acid receptors in the process (Hubbard et al., 2010). Abscisic acid receptor PYL was one of the receptors, and CEY00_Acc03316 was annotated as abscisic acid receptor PYL, so it could be inferred that CEY00_Acc03316 may be a cold tolerance gene. The overexpression of bZIP transcription factor family proteins under stress was reported to improve the cold tolerance of plants (Chang et al., 2017; Ma et al., 2018). Therefore, CEY00_Acc13130 gene may be a cold tolerance gene. TIFY protein was induced and expressed at low temperature, and its gene family might be involved in low-temperature response and adaptation (Ye et al., 2009; Wang et al., 2017). Therefore, it could be inferred that CEY00_Acc33627 gene may be a cold tolerance gene.

Starch and sucrose metabolic pathway was found to be related to cold tolerance (Li et al., 2011, 2018). So the DEGs of starch and sucrose metabolism pathway were analyzed (Figure 4). There were eight DEGs in starch and sucrose metabolism pathway, of which six were CEY00_Acc26744 (alphatrehalose phosphate synthase), CEY00_Acc28966 (beta-amylase), CEY00_Acc16695 (beta-amylase), CEY00_Acc14271 (beta-glucosides GH1 family), CEY00_Acc16756 (trehalose phosphatase) and CEY00_Acc08918 (beta-amylase). There were two down-regulated DEGs, CEY00_Acc17108 (betaglucosidase) and CEY00_Acc04508 (alpha-amylase) (Table 9).

Trehalose-6-phosphate synthase can protect biological cell membrane and protein from damage, and is widely used in transgenic experiments with plants for the improvement of cold tolerance of plants (Kim et al., 2017). CEY00_Acc26744 and CEY00_Acc16756 were annotated as alpha-trehalosephosphate synthase genes and inferred as cold-resistant genes. Maltose, which is produced by starch decomposition catalyzed by beta-amylase, may help protect the electron transport chain and protein in the stress environment. Cold tolerance of plants could be regulated by soluble sugar (Tarkowski and Van den Ende, 2015). Beta-amylase might reduce cold stress injury by increasing the content of sugars and enhance the cold tolerance of plants (Lv et al., 2017). Hence, unigenes of CEY00_Acc28966 (beta-amylase), CEY00_Acc16695 (beta-amylase) and CEY00_Acc08918 (beta-amylase) were inferred as cold-resistant genes.

\section{RT-qPCR validation}

The results RT-qPCR analysis of selected DEGs showed that the expression levels of all the genes selected were consistent with the transcriptome results (Figure 5). The findings suggested that there were statistically significant differences between the expression levels of these genes in low-temperature treated and control plantlets.

\section{Discussions}

The methods of inducing low-temperature stress in plants are mainly in the low-temperature environments, such as low-temperature natural environment, temperature control box or low-temperature refrigerator for direct screening. For example, Yuan et al. (1996) induced the natural low-temperature in Robinia pseudoacacia for 3 years, which improved the root cold tolerance of $R$. pseudoacacia. Chemical reagents can also be used as a selection pressure for stress and screening, in which L-Hyp is often used as a selection pressure in the study of plant cold tolerance. L-Hyp is a proline analog, which has an inhibitory effect on plants. It is often used as a selective pressure to screen all kinds of stress tolerance of plants, such as salt tolerance, cold tolerance, drought tolerance and so on (Chen et al., 1995; Gong et al., 2012). L-Hyp is often used in low-temperature stress experiments, but also can be used as a cold-resistant mutagen. Peng et al (2014) induced ornamental pineapple with EMS and screened for tolerance to HYP to induce coldresistant mutants. In this study, L-Hyp selective pressure and low-temperature stress were used to screen the cold-resistant kiwifruit plantlets, and these two methods were found to be effective.

Janská et al. (2011) found that plant signal transduction pathway plays a key role in the response of plants to low temperature. In this study, it was found that the DEGs enriched in plant hormone signal transduction pathway were related to cold tolerance, which was consistent with the results of Janská et al. (2011). The starch metabolic pathway was reported to be closely related to the cold tolerance response of plants (Li et al., 2018). In this study, the genes of the plant hormone signal transduction, and starch and sucrose metabolism pathway were found to be up-regulated in the treatment group, including CEY00_Acc03316 (abscisic acid receptor PYL), CEY00_Acc13130 (bZIP transcription factor), CEY00_Acc33627 (TIFY protein). 
CEY00_Acc26744 (alpha-trehalose-phosphate synthase), CEY00_Acc16756 (trehalose phosphatase), CEY00_Acc28966 (beta-amylase), CEY00_Acc16695 (beta-amylase), and CEY00_Acc08918 (beta-amylase). ABA has a certain effect on improving the cold tolerance in plants (Li et al., 2019). Therefore, the differentially expressed gene CEY00_Acc03316 (abscisic acid receptor PYL) might play an important role in the improvement of plant cold tolerance. BZIP transcription factors were found to play an important role in regulating plant cold tolerance and other stresses (Banerjee and Roychoudhury, 2017). Hence, CEY00_Acc13130 (bZIP transcription factor) might be a cold-tolerance related gene. Trehalose-6-phosphate synthase, a key enzyme in trehalose synthesis, was found to play an important role in the low-temperature response in different plant species (Miranda et al., 2007; Ge et al., 2008). Therefore, alpha-trehalose-phosphate synthase (CEY00_Acc26744) identified in the study, might be a cold-tolerance related gene. Betaamylase affected the cold-tolerance of plants mainly by regulating the content of starch (Peng et al., 2014). Beta-amylase-mediated starch degradation was found to play an important role in cold tolerance of plants (Lv et al., 2017). So beta-amylase (CEY00_Acc28966, CEY00_Acc16695 and CEY00_Acc08918) might be important cold tolerance genes in the kiwifruit.

At present, the researches on kiwifruit transcriptome analysis are mainly focused on the changes of fruit pulp color, fruit pigment, fruit development, bacterial canker and so on (Asiche et al., 2018; Song et al., 2019). There were few studies on the stress tolerance of kiwifruit transcriptome analysis, especially on cold tolerance, and there was no study on cold-resistant genes of kiwifruit, which needs to be studied in the future. The genes related to cold tolerance identified in the study need further functional verification.

\section{Conclusions}

The tetraploid plantlets were treated and screened by L-Hyp selective pressure and low-temperature stress, and the cold-tolerance of kiwifruit was determined by using growth indexes. The highest tolerance of tetraploidy to low-temperature treatment was $0{ }^{\circ} \mathrm{C}$ for $12 \mathrm{~h}$, and the survival rate was $42.22 \%$. A total of 126 cold-resistant plantlets were obtained.

Based on the transcriptome analysis of tetraploid cold-resistant plants after low-temperature treatment, two pathways related to cold tolerance were obtained, which were plant hormone signal transduction and starch and sucrose metabolism pathway. In these two pathways, a total of 8 DEGs of coldtolerance in kiwifruit were screened. And these genes were confirmed to be up-regulated by RT-qPCR when the tetraploid plantlets were treated with cold temperature.

\section{Methods}

\section{Materials}

The experimental materials were diploid plants of yellow kiwifruit variety 'SWFU03' from the Plant Nursery, Southwest Forestry University and its tetraploid plants which were induced and identified by Li et al. (2019). In-vitro propagated tetraploid and diploid plants come from three mother tetraploid plants and three diploid plants. Medium, grow chamber conditions, and photoperiod of the plantlets were described previously by Li et al. (2019).

\section{Screening of cold-resistant mutants in tetraploidy}

\section{Screening for cold-tolerant mutants with L-Hyp}

The tissue culture plantlets of purified kiwifruit tetraploidy were propagated. The tetraploid tissue culture plantlets of $A$. chinensis, whose ploidy had been identified as tetraploidy, were inserted into the basic medium supplemented with L-Hyp at different concentrations ( $0 \mathrm{mmol} / \mathrm{L}, 2 \mathrm{mmol} / \mathrm{L}, 4 \mathrm{mmol} / \mathrm{L}, 6$ $\mathrm{mmol} / \mathrm{L}, 8 \mathrm{mmol} / \mathrm{L}$ and $10 \mathrm{mmol} / \mathrm{L}$ ). The survival rate, fresh weight of plantlets, the total number of leaves and the height of plantlets were measured before and after treatment. The culture temperature was $(25 \pm 3)^{\circ} \mathrm{C}$, and the changes of these indexes were observed after 30 days.

At the same time, the diploid tissue culture plantlets of $A$. chinensis were inserted into the basic medium supplemented with L-Hyp at different concentrations $(0 \mathrm{mmol} / \mathrm{L}, 2 \mathrm{mmol} / \mathrm{L}, 4 \mathrm{mmol} / \mathrm{L}, 6 \mathrm{mmol} / \mathrm{L}, 8 \mathrm{mmol} / \mathrm{L}$ and $10 \mathrm{mmol} / \mathrm{L})$. There were 5 bottles for each concentration, and one tissue culture seedling was inoculated in one bottle. The survival rate and fresh weight of plantlets, the total number of leaves and the height of plantlets were measured before and after treatment. The changes of indexes were observed after being cultured in the same environment for 7 days. Then the plantlets were transferred to the normal medium without L-Hyp for 30 days, repeated three times, to screen the cold-tolerant diploid kiwifruit plantlets.

\section{Directional screening of cold-resistant mutants by low temperature treatment}

Yellow kiwifruit diploid plantlets and tetraploid plantlets identified were used as experimental materials. The plantlets were treated with low temperature at $0{ }^{\circ} \mathrm{C}$ for $3 \mathrm{~h}, 5 \mathrm{~h}, 8 \mathrm{~h}, 12 \mathrm{~h}$ and $24 \mathrm{~h}$, respectively ( $\mathrm{n}=15$, repeated 3 times). The aseptic diploid plantlets were treated with the same time as the tetraploidy. The wilting degree, survival rate and mortality of aseptic tetraploid and diploid plantlets were measured.

\section{Tetraploidy transcriptome analysis of yellow kiwifruit}

\section{Test materials and methods}

Three bottles of tetraploid plantlets were treated at $0{ }^{\circ} \mathrm{C}$ for $5 \mathrm{~h}\left(3\right.$ plantlets in each bottle) and 3 bottles of tetraploid plants treated at $25^{\circ} \mathrm{C}$ for $5 \mathrm{~h}(3$ plantlets in each bottle). The bottles were placed on a clean table. In the normal temperature control groups (C1, C2 and C3) and low-temperature treatment groups (T1, T2 and T3), three plantlets (1 plant per bottle) were packed and labeled with tin foil. After sampling, the samples were quickly 
frozen in cryogenic liquid nitrogen and then placed in dry ice. Six samples were sent to Anhui Microanalysis Gene Technology Co., Ltd for transcriptome analysis. RNA-Seq was performed using Illumina HiSeqTM 2500, and RNA-Seq data were presented at the Genome Sequence Archive of the Beijing Institute of Genomics (BIG) Data Center (accession number CRA001986).

The data produced by the RNA-Seq sequence were compared to the reference genome of $A$. chinensis. The differences between samples were based on the results of quantitative expression, and Gene Ontology (GO) and Kyoto Encyclopedia of Genes and Genomes (KEGG) pathway analysis of the differential genes carried out in the study.

\section{Sequence data filtering}

After the library was constructed, the Illumina HiSeqTM 2500 platform was used for sequencing, and the sequence strategy was PE150. The original data (raw reads) obtained by sequencing were filtered, and the low-quality reads, connectors, and contamination, which accounted for more than $40 \%$ of the total read, were filtered out of the alkali base with a mass value of less than 15.

\section{Comparison of reads obtained by sequencing with the reference genome}

After the clean reads were obtained, the clean reads were compared to the whole genome sequence of $A$. chinensis by HISAT2, and the reads mapping information was obtained. The utilization rate of sequence data and the genetic relationship with the whole genome of $A$. chinensis were obtained by comparison.

\section{Detection of differentially expressed genes (DEGs)}

The screening threshold was FDR (false discovery rate) $<0.05$, log2FC (fold change for a gene) $>1$ or log2FC $<-1$. Based on the results of expression analysis, read count information and DEseq2 (or edgeR) software were used to analyze DEGs (Anders and Huber, 2010).

\section{Enrichment analysis of DEGs in GO category and KEGG Pathway}

The DEGs were annotated on the Gene Ontology database (GO, http://www.geneontology.org/), and the GO term with FDR $\leq 0.05$ was selected as the significantly enriched GO entry (Anders and Huber, 2010). The DEGs were annotated on the KEGG Pathway database (Liu et al., 2018).

\section{Screening of differential genes}

Differential gene data were annotated to GO and KEGG were analyzed, and the cold tolerance related genes in kiwifruit were screened according to the previous studies on cold tolerance genes and related pathways (Hubbard et al., 2010; Kim et al., 2017; Li et al., 2018; Wang et al., 2017; Ma et al., 2018).

\section{RT-qPCR analysis}

The transcriptional expression levels of 3 genes in the pathway of plant hormone signal transduction and 7 genes in the pathway of starch and sucrose metabolism pathway were selected for checking by RT-qPCR in low-temperature and room-temperature treated plantlets. The total RNA was extracted using the Qiagen RNeasy Mini Kit (Qiagen Inc., Valencia, CA), and then reversely transcribed into cDNA by random primers. The RT-qPCR analysis was conducted according to a previous report (Liu et al., 2018). Gene-specific primers were designed using Primer Premier 5.0 software, and the primers used for RT-qPCR analyses were listed in Table1. The2 $(-\triangle \Delta$ Ct) method (Liu et al., 2018) was used to analyze the data.

\section{Abbreviations}

BIG: Beijing institute of genomics; DEGs: differentially expressed genes; FC: fold change for a gene; FDR: false discovery rate; GO: gene ontology; KEGG: kyoto encyclopedia of genes and genomes; L-Hyp: L-hydroxyproline; RT-qPCR: real time quantitative polymerase chain reaction.

\section{Declarations}

\section{Ethics approval and consent to participate}

The experiment materials does not include human being or animal. Hence, ethics approval and consent to participate is not applicable.

\section{Consent for Publication}

Not applicable.

\section{Availability of data and material}

All data generated or analysed during this study are included in this published article. RNA-Seq data were presented at the Genome Sequence Archive of the Beijing Institute of Genomics (BIG) Data Center (accession number CRA001986).

\section{Competing interests}


The authors declare that they have no competing interests.

\section{Funding}

The project was support from the National key R \& D Plan for the 13th Five-Year Plan Project of China (Grant No. 2016YFD0600102), the National Natural Science Foundation of China (Grant No. 31760450), and the Fund for the Introduction of Foreign Talents and Intelligence of Yunnan Province (Grant No.YN2018010). The funders had no role in the design of the study and collection, analysis, and interpretation of data and in writing the manuscript.

\section{Author contributions}

$\mathrm{HZ}$ conceived and designed the experiments. YL, $\mathrm{XL}$ and ZW performed the experiments. $\mathrm{YL}, \mathrm{SL}$ and $\mathrm{XL}$ analyzed the data, and wrote the paper. ZZ and WB contributed analysis tools. XL edited the paper. All authors have read and approved the manuscript.

\section{Acknowledgments}

The authors thank Research Scientist Xiuying Chen, the New Zealand Institute for Plant \& Food Research for her critical reading of the manuscript. And the manuscript was proofread by Proofed Inc. (UK).

\section{References}

Anders S, \& Huber W. Differential expression analysis for sequence count data. Gene Biology (2010)11: R106.

Asiche WO, Mitalo OW, Kasahara Y, Tosa Y, Mworia EG, Owino WO, Ushijima K, Nakano R, Yano K, \& Kubo Y. Comparative transcriptome analysis reveals distinct ethylene-independent regulation of ripening in response to low temperature in kiwifruit. BMC Plant Biology (2018) $18(1): 47$.

Banerjee A, \& Roychoudhury A. Abscisic-acid-dependent basic leucine zipper (bZIP) transcription factors in plant abiotic stress. Protoplasma (2017) 254(1):3-16.

Chang Y, Nguyen BH, Xie Y, Xiao B, Tang N, Zhu W, Mou T, \& Xiong L. Co-overexpression of the constitutively active form of OsbZIP46 and ABA-activated protein kinase SAPK6 improves drought and temperature stress resistance in rice. Frontiers in Plant Science (2017) 8:1102.

Chen H, Kuang B, \& Wang J. Studies on selection and characterization of a stress-tolerant sugarcane cell line. Chinese Journal of Biotechnology (1995) 11(2):93-99.

Ge LF, Chao DY, Shi M, Zhu MZ, Gao JP, \& Lin HX. Overexpression of the trehalose-6-phosphate phosphatase gene OsTPP1 confers stress tolerance in rice and results in the activation of stress responsive genes. Planta (2008) 228(1):191-201.

Gong SY, Huang GQ, Sun X, Li P, Zhao LL, Zhang DJ, \& Li XB. GhAGP31, a cotton non-classical arabinogalactan protein, is involved in response to cold stress during early seedling development. Plant Biology (2012) 14(3):447-57.

Hubbard KE, Nishimura N, Hitomi K, Getzoff ED, \& Schroeder Jl. Early abscisis acid signal transduction mechanisms: newly discovered components and newly emerging questions. Genes Development (2010) 24:1695-1708.

Janská A, Marsík P, Zelenková S, \&Ovesná J. Cold stress and acclimation - what is important for metabolic adjustment? Plant Biology (2010) 12(3):395405 .

Kim Y, Lee DW, \& Jung JK. Rapid Cold-hardening of a subtropical species, Maruca vitrata (Lepidoptera: Crambidae), accompanies hypertrehalosemia by upregulating trehalose-6-phosphate synthase. Environmental Entomology (2017) 6(6):1432-1438.

Li S, Liu X, Liu H, Zhang X, Ye Q, Zhang H. Induction, identification and genetics analysis of tetraploid Actinidia chinensis. Royal Society Open Science (2019) 6: 191052.

Li S, Yang Y, Zhang Q, Liu N, Xu Q, \& Hu L. Differential physiological and metabolic response to low temperature in two zoysiagrass genotypes native to high and low latitude. PLoS One (2018) 13(6): e0198885.

Li T, Xu SL, Oses-Prieto JA, Putil S, Xu P, Wang RJ, Li KH, Maltby DA, An LH, Burlingame AL, Deng ZP, \& Wang ZY. Proteomics analysis reveals posttranslational mechanisms for cold-induced metabolic changes in Arabidopsis. Molecular Plant (2011) 4(2):361-74.

Li Y, Wang X, Ban Q, Zhu X, Jiang C, Wei C, \&Bennetzen JL. Comparative transcriptomic analysis reveals gene expression associated with cold adaptation in the tea plant Camellia sinensis. BMC Genomics (2019) 20(1):624.

Liu X, Zhang Z, Sang M, Sun X, He C, Xin P, \& Zhang H. Functional analysis of the FZF1 gene in Saccharomyces uvarum. Frontiers in Microbiology(2018) 9, 96. 
Lv Y, Yang M, Hu D, Yang Z, Ma S, Li X, \&Xiong L. The OsMYB30 Transcription factor suppresses cold tolerance by interacting with a JAZ protein and suppressing $\beta$-Amylase expression. Plant Physiology (2017) 173(2):1475-1491.

Ma H, Liu C, Li Z, Ran Q, Xie G, Wang B, Fang S, Chu J, \& Zhang J. ZmbZIP4 contributes to stress resistance in maize by regulating ABA synthesis and root development. Plant Physiology (2018) 178(2):753-770.

Minchin PEH, De Silva N, Snelgar WP, \& Richardson

AC. Modelling of colour development in the fruit of Actinidia chinensis 'Hort16A'. New Zealand Journal of Crop and Horticultural Science (2003) 31(1):4153.

Miranda JA, Avonce N, Suárez R, Thevelein JM, Van Dijck P, \&lturriaga G. A bifunctional TPS-TPP enzyme from yeast confers tolerance to multiple and extreme abiotic-stress conditions in transgenic Arabidopsis. Planta (2007) 226(6):1411-21.

Peng T, Zhu X, Duan N, \& Liu JH. PtrBAM1, a $\beta$-amylase-coding gene of Poncirus trifoliata, is a CBF regulon member with function in cold tolerance by modulating soluble sugar levels. Plant Cell and Environment (2014) 37(12), 2754-2767.

Song Y, Sun L, Lin M, Chen J, Qi X, Hu C, \& Fang J. Comparative transcriptome analysis of resistant and susceptible kiwifruits in response to Pseudomonas syringae pv. Actinidiae during early infection. PLoS One (2019) 14(2):e0211913.

Tarkowski ŁP, \& Van den Ende W. Cold tolerance triggered by soluble sugars: a multifaceted countermeasure. Frontiers in Plant Science (2015) 6:203.

Vallespir F, Rodríguez Ó, Cárcel JA, Rosselló C, \&Simal S. Ultrasound assisted low-temperature drying of kiwifruit: Effects on drying kinetics, bioactive compounds and antioxidant activity. Journal of Science Food Agriculture (2019) 99(6):2901-2909.

Wang Y, Pan F, Chen D, Chu W, Liu H, \& Xiang Y. Genome-wide identification and analysis of the Populus trichocarpa TIFY gene family. Plant Physiology Biochemistry (2017) 115:360-371.

Wu JH, Ferguson AR, \& Murray BG. Manipulation of ploidy for kiwifruit breeding: in vitro chromosome doubling in diploid Actinidia chinensis Planch. Plant Cell Tissue and Organ Culture (2011) 106(3):503-511.

Wu JH, Ferguson AR, Murray BG, Jia Y, Datson PM, \& Zhang J. Induced polyploidy dramatically increases the size \& alters the shape of fruit in Actinidia chinensis. Annals of Botany(2012) 109(1):169.

Wu JH, Ferguson AR, Murray BG, Geijskes RJ, Lakshmanan P, \& Taji A. In vitro induction of autotetraploid Actinidia plants and their field evaluation for crop improvement. Acta Horticulture (2009): 245-250.

Ye H, Du H, Tang N, Li X, \&Xiong L. Identification and expression profiling analysis of TIFY family genes involved in stress and phytohormone responses in rice. Plant Molecular Biology (2009) 71(3):291-305.

Yuan J, \& Wang L. Survival of Escherichia coli 0157:H7, Salmonella spp., and Listeria monocytogenes on Fresh and Sliced Green and Golden Kiwifruits. Foodborne Pathogens and Disease (2018) 15(9):560-567.

Yuan YX, Wang Y, Pei BH, \& Jia XG. Effect of cold inducement on hardiness of Amorpha fruticosa and Robinia pseudoacacia. Chinese Journal of Plant Ecology (1996) 20(1):65- 73. (In Chinese)

Zhang HY, Liu HM, \&Liu XZ. Production of transgenic kiwifruit plants harboring the SbtCry1Ac gene. Genetics and Molecular Research (2015) 14 (3): 8483.

\section{Tables}

Table 1. Primer sequences of target genes (TG) and reference genes (RG) used in RT-qPCR. 


\begin{tabular}{|c|c|c|c|}
\hline Gene name & Primer & Sequence $\left(5^{\prime}-3^{\prime}\right)$ & Length (bp) \\
\hline \multirow[t]{2}{*}{ Elongation factor (RG) } & Forward primer & ACAAGCTGGTGACAATGTGG & 127 \\
\hline & Reverse primer & CGACCACCTTCATCCTTTGT & \\
\hline Abscisic acid receptor PYL & Forward primer & & 149 \\
\hline \multirow[t]{3}{*}{ (CEY00_Acc03316, TG) } & & GGTTTGGGAGGCTACTGAGT & \\
\hline & Reverse primer & & \\
\hline & & TCCATTCGCATTCATCGCTG & \\
\hline \multirow[t]{4}{*}{ BZIP transcription factor(CEY00_Acc13130,TG) } & Forward primer & & 332 \\
\hline & & TGTTTCTTGTGGATTGGCGG & \\
\hline & Reverse primer & & \\
\hline & & TGCCCCATGTAGTTTCCCAT & \\
\hline \multirow[t]{4}{*}{ TIFY protein(CEY00_Acc33627, TG) } & Forward primer & & 237 \\
\hline & & ATCСССТGACССТСССТАТТ & \\
\hline & Reverse primer & & \\
\hline & & CTCCGGGTTCATCTTCGAGA & \\
\hline \multirow[t]{4}{*}{ Alpha-trehalose-phosphate synthase(CEY00_Acc26744, TG) } & Forward primer & & 225 \\
\hline & & TCGTCGGGGAATGATGATGT & \\
\hline & Reverse primer & & \\
\hline & & GCATTCGATCAAACGGGTCA & \\
\hline \multirow[t]{4}{*}{ Beta-amylase (CEY00_Acc28966, TG) } & Forward primer & & 226 \\
\hline & & ATGCTTGGTGGGGATTGGTA & \\
\hline & Reverse primer & & \\
\hline & & GCCCGATCTGTCTGTGTAGA & \\
\hline \multirow[t]{4}{*}{ Beta-glucosidase 1 GH1 family(CEY00_Acc14271, TG) } & Forward primer & & 201 \\
\hline & & GGGCCTCGGTGAAGTTTTAC & \\
\hline & Reverse primer & & \\
\hline & & CCCCTTGATGTTGACTCCCT & \\
\hline \multirow[t]{4}{*}{ Trehalose phosphatase(CEY00_Acc16756, TG) } & Forward primer & & 160 \\
\hline & & CGGTTGCGACTAACTCATGG & \\
\hline & Reverse primer & & \\
\hline & & GCATCTTCGTCGGTCTTGTC & \\
\hline \multirow{4}{*}{ Beta-amylase 4 (CEY00_Acc08918, TG) } & Forward primer & & 149 \\
\hline & & CTTGGAGATGGCGAAGAAGC & \\
\hline & Reverse primer & & \\
\hline & & TCTGTGTAGGCAAGGTCAGG & \\
\hline \multirow[t]{2}{*}{ Beta-glucosidase 12-like (CEY00_Acc17108, TG) } & Forward primer & & 155 \\
\hline & & CCAAATTCACACCCGAGCAA & \\
\hline
\end{tabular}


TTACCGAGgTGAGATTGGCA

Alpha-amylase (CEY00_Acc04508, TG)

Forward primer

286

ACAGGATCAACACAGGCTCA

Reverse primer

ATCGGCTGTTGAGgtCTTGA

Table 2. Plant survival rates in tetraploid and diploid plantlets treated with L-Hyp.

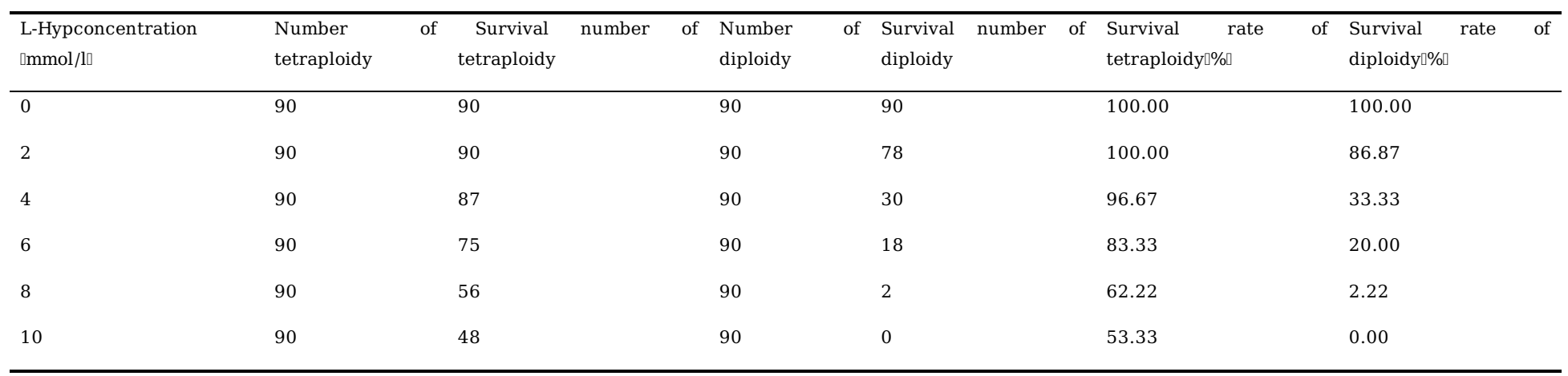

Note: The data in the table were measured after 30 days of treatment.

Table3 Changes in fresh weight of plants under different L-Hyp concentrations.

\begin{tabular}{|c|c|c|c|c|c|c|}
\hline $\begin{array}{l}\text { L-Hypconcentration } \\
\square \mathrm{mmol} / \square\end{array}$ & $\begin{array}{lr}\text { Fresh weight } & \text { of } \\
\text { tetraploidy } & \text { before } \\
\text { treatment }(\mathrm{g}) & \end{array}$ & $\begin{array}{lr}\text { Fresh weight } & \text { of } \\
\text { tetraploidy } & \text { after } \\
\text { treatment }(\mathrm{g}) & \end{array}$ & $\begin{array}{l}\text { Fresh weight of diploidy } \\
\text { before treatment }(\mathrm{g})\end{array}$ & $\begin{array}{llr}\text { Fresh weight } & \text { of } \\
\text { diploidy } & & \text { after } \\
\text { treatment } & (\mathrm{g}) & \end{array}$ & $\begin{array}{l}\text { Increased rate of } \\
\text { tetraploidy (\%) }\end{array}$ & $\begin{array}{l}\text { Increased rate } \\
\text { of diploidy (\%) }\end{array}$ \\
\hline 0 & 0.769 & 0.852 & 0.686 & 0.783 & 110.79 & 114.13 \\
\hline 2 & 0.651 & 0.678 & 0.738 & 0.691 & 104.14 & 93.63 \\
\hline 4 & 0.515 & 0.525 & 0.551 & 0.365 & 101.94 & 66.24 \\
\hline 6 & 0.726 & 0.729 & 0.817 & 0.408 & 100.41 & 49.93 \\
\hline 8 & 0.821 & 0.795 & 0.973 & 0.100 & 96.83 & 10.28 \\
\hline 10 & 0.674 & 0.510 & 0.712 & 0.000 & 75.67 & 0.00 \\
\hline
\end{tabular}

Table 4. Height of seedlings treated with L-Hyp at different concentrations. 


\begin{tabular}{|c|c|c|c|c|c|c|c|}
\hline $\begin{array}{l}\text { L-Hypconcentration } \\
\square \mathrm{mmol} / \mathrm{l} \square\end{array}$ & $\begin{array}{l}\text { Height of } \\
\text { tetraploidy } \\
\text { seedling before } \\
\text { treatment of }(\mathrm{cm})\end{array}$ & $\begin{array}{l}\text { Height of tetraploidy } \\
\text { seedling after treatment } \\
\text { of } \\
\text { (cm) }\end{array}$ & $\begin{array}{l}\text { Height of } \\
\text { seedling } \\
\text { treatment of } \\
\text { (cm) }\end{array}$ & $\begin{array}{r}\text { diploidy } \\
\text { before }\end{array}$ & $\begin{array}{l}\text { Height of diploidy seedling } \\
\text { after treatment of }(\mathrm{cm})\end{array}$ & $\begin{array}{l}\text { Growth rate of } \\
\text { tetraploidy }(\%)\end{array}$ & $\begin{array}{l}\text { Growth rate of } \\
\text { diploidy }(\%)\end{array}$ \\
\hline 0 & 1.72 & 2.41 & 1.87 & & 2.86 & 140.12 & 152.94 \\
\hline 2 & 1.64 & 1.98 & 1.73 & & 1.82 & 120.73 & 105.20 \\
\hline 4 & 1.73 & 2.03 & 1.57 & & 1.60 & 117.51 & 101.91 \\
\hline 6 & 2.15 & 2.18 & 2.06 & & 1.98 & 101.40 & 96.17 \\
\hline 8 & 1.98 & 2.12 & 1.95 & & 1.56 & 101.62 & 80.00 \\
\hline 10 & 1.81 & 1.91 & 1.73 & & 0.00 & 105.52 & 0.00 \\
\hline
\end{tabular}

Table 5. Survival rate of diploid and tetraploid plantlets under low-temperature stress.

\begin{tabular}{|c|c|c|c|c|}
\hline Treatment & Survival rate of tetraploidyø $\%$ & Survival rate of diploidy $\square$ & $\begin{array}{l}\text { Dead rate of tetraploidy } \\
\square \%\end{array}$ & $\begin{array}{l}\text { Dead rate of diploidy } \\
\square \%\end{array}$ \\
\hline $0^{\circ} \mathrm{C}, 3 \mathrm{~h}$ & 88.89 & 17.78 & 11.11 & 82.22 \\
\hline $0^{\circ} \mathrm{C}, 5 \mathrm{~h}$ & 80.00 & 4.44 & 20.00 & 95.56 \\
\hline $0^{\circ} \mathrm{C}, 8 \mathrm{~h}$ & 68.89 & 2.22 & 31.11 & 97.78 \\
\hline $0^{\circ} \mathrm{C}, 12 \mathrm{~h}$ & 42.22 & 0.00 & 44.44 & 100.00 \\
\hline $0^{\circ} \mathrm{C}, 24 \mathrm{~h}$ & 0.00 & 0.00 & 100 & 100.00 \\
\hline
\end{tabular}

Table 6. Raw data and clean data output statistics.

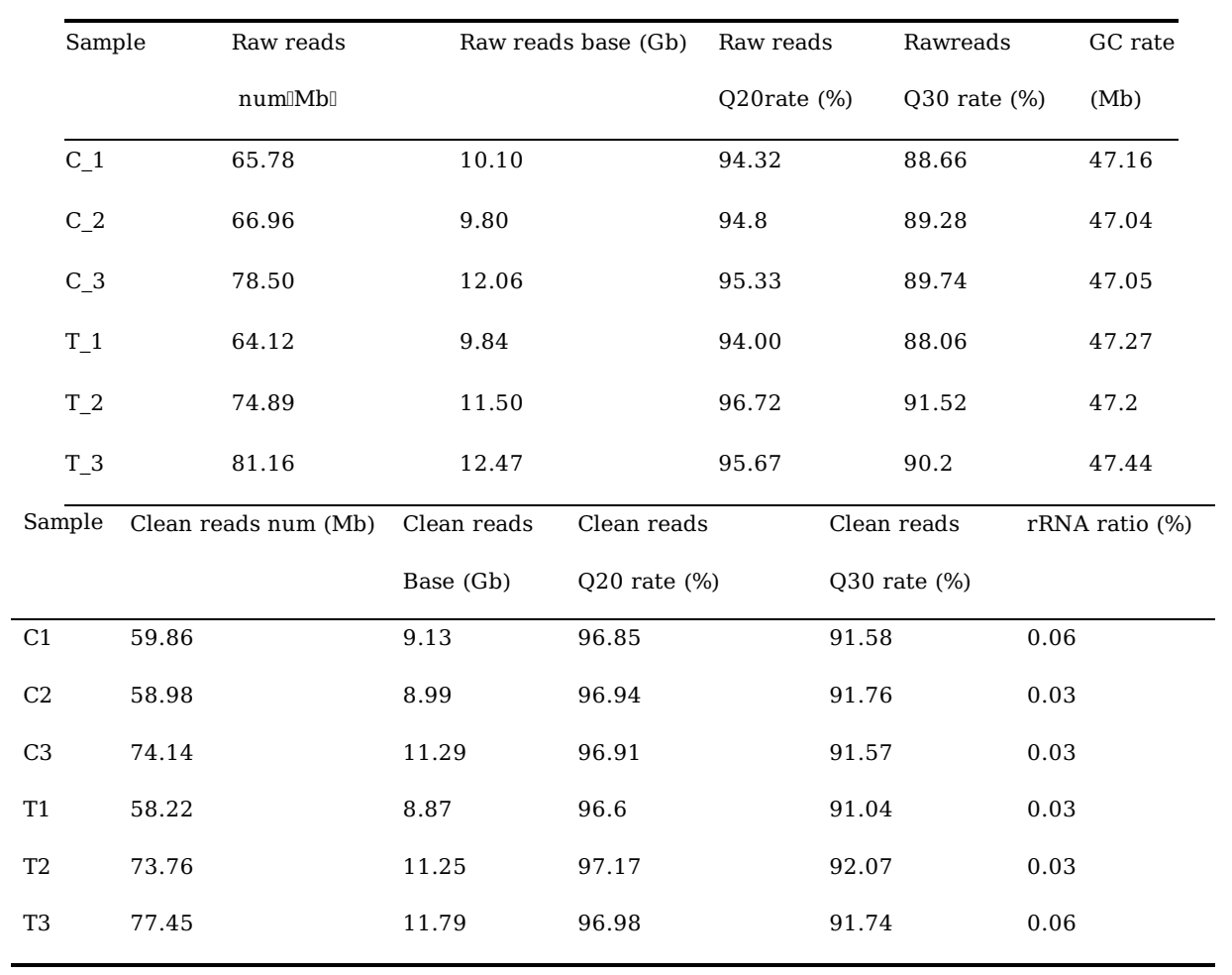


Table 7. Statistical groups of differentially expressed genes in all comparison groups.

\begin{tabular}{lll}
\hline Group & \multicolumn{2}{c}{ Total Up } \\
\hline C-vs-T & 16306191011 \\
C1-vs-T1 169 & 81 & 88 \\
C2-vs-T2 281 & 133148 \\
C3-vs-T3 836 & 274562 \\
\hline
\end{tabular}

Group, differential expression group; Total, the number of all DEGs; Up, the number of up-regulated DEGs; Down, the number of down-regulated DEGs.

Table 8. Analysis of differentially expressed genes in plant hormone signal transduction and enrichment.

\begin{tabular}{|c|c|c|c|c|}
\hline Gene ID & Gene length & Gene name & $\log 2(\mathrm{FC})$ & Regulation \\
\hline CEY00_Acc07445 & 648 & Pathogenesis-related leaf protein & 5.28215967 & Up \\
\hline CEY00_Acc03316 & 978 & Abscisic acid receptor PYL & 2.41039795 & Up \\
\hline CEY00_Acc10294 & 2193 & Probable indole-3-acetic acid-amido synthetase GH3 & 1.78757476 & Up \\
\hline CEY00_Acc13130 & 1934 & bZIP transcription factor family protein & 1.63993265 & Up \\
\hline CEY00_Acc33627 & 1598 & Protein TIFY & 1.41856611 & Up \\
\hline CEY00_Acc21162 & 1191 & Pathogenesis-related protei & 1.38666594 & Up \\
\hline CEY00_Acc11766 & 1492 & Cyclin D3-1 & -1.36287325 & Down \\
\hline CEY00_Acc06865 & 671 & Basic form of Pathogenesis-related protein & -1.38277358 & Down \\
\hline CEY00_Acc07128 & 1837 & Amino acid transporter & -1.48608531 & Down \\
\hline CEY00_Acc19743 & 469 & Hypothetical protein CICLE & -1.51643111 & Down \\
\hline CEY00_Acc06866 & 679 & Basic form of pathogenesis-related protein & -1.52680253 & Down \\
\hline CEY00_Acc13744 & 2123 & Indole-3-acetic acid-amido synthetase GH3.6 & -1.67570429 & Down \\
\hline CEY00_Acc16267 & 524 & Hypothetical protein CICLE & -1.72359172 & Down \\
\hline CEY00_Acc07415 & 2003 & Indole-3-acetic acid-amido synthetase GH3.1 & -1.74567531 & Down \\
\hline CEY00_Acc04564 & 706 & Auxin early response protein SAUR & -1.75092162 & Down \\
\hline CEY00_Acc21097 & 1536 & Cyclin-D3-1 & -1.75509953 & Down \\
\hline CEY00_Acc10793 & 1871 & Auxin transporter-like protein & -1.76951247 & Down \\
\hline CEY00_Acc10304 & 1525 & Cyclin D3-2 & -1.95276355 & Down \\
\hline CEY00_Acc32114 & 1035 & Hypothetical protein VITISV & -2.16269734 & Down \\
\hline CEY00_Acc26372 & 2067 & GH3 auxin-responsive promoter & -2.50991627 & Down \\
\hline CEY00_Acc04566 & 1001 & Auxin-induced protein 6B-like & -2.75593648 & Down \\
\hline CEY00_Acc28473 & 851 & Auxin-responsive protein IAA & -2.79532550 & Down \\
\hline CEY00_Acc23775 & 1717.72 & Cyclin_N domain-containing protein & -2.82746855 & Down \\
\hline CEY00_Acc03517 & 1315 & Hypothetical protein CICLE & -3.74278320 & Down \\
\hline CEY00_Acc07283 & 736 & Auxin-induced protein 15A-like & -4.08073735 & Down \\
\hline
\end{tabular}


Table 9. Analysis of DEGs in starch and sucrose metabolic pathways.

\begin{tabular}{lllll}
\hline Gene ID & Gene length (bp) & Gene name & $\log 2(\mathrm{FC})$ & Regulation \\
\hline CEY00_Acc26744 & 2652 & Alpha-trehalose-phosphate synthase & 3.12212772 & Up \\
CEY00_Acc28966 & 1926 & Beta-amylase & 2.16097859 & Up \\
CEY00_Acc16695 & 1841 & Beta-amylase & 1.82925763 & Up \\
CEY00_Acc14271 & 1766 & Beta-glucosidase 1 GH1 family & 1.59187712 & Up \\
CEY00_Acc16756 & 1833 & Trehalose-phosphate phosphatase F & 1.49443763 & Up \\
CEY00_Acc08918 & 1989 & Beta-amylase 4 & 1.34221944 & Up \\
CEY00_Acc17108 & 1465 & Beta-glucosidase 12-like & -1.38324393 & Down \\
CEY00_Acc04508 & 1435 & Alpha-amylase & -2.92269962 & Down \\
\hline
\end{tabular}

\section{Figures}

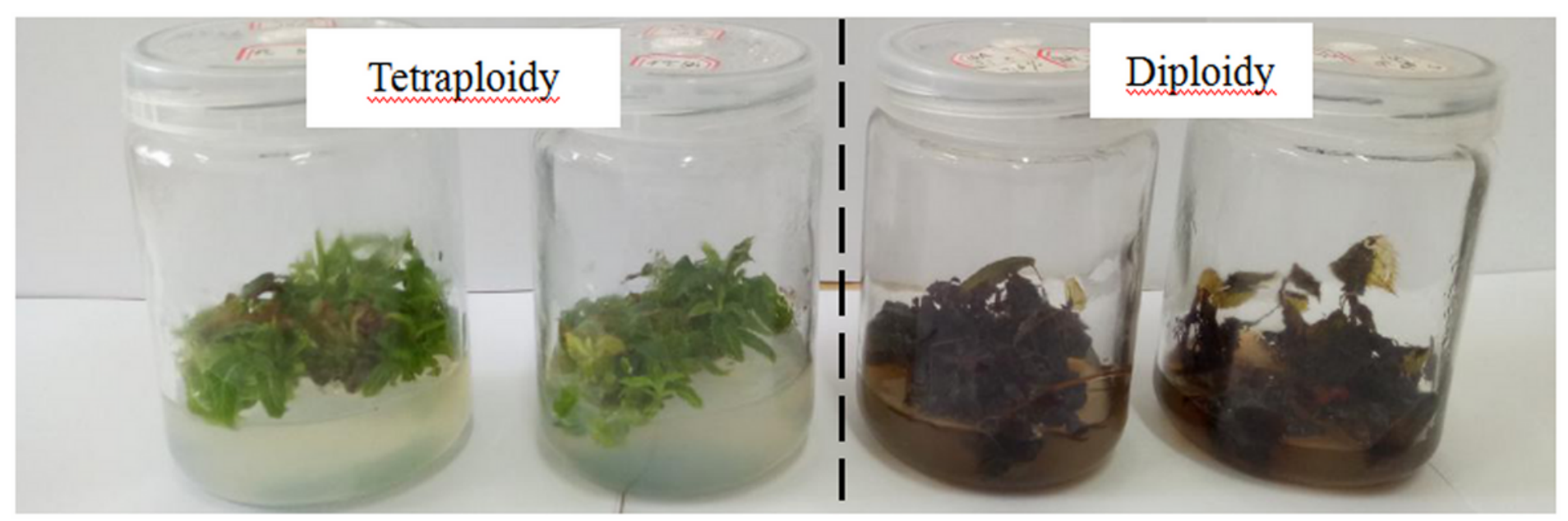

\section{Figure 1}

Changes of tetraploid and diploid plantlets under low-temperature treatment. The plantlets were placed at room temperature for 14 days after lowtemperature treatment at $0{ }^{\circ} \mathrm{C}$ for $12 \mathrm{~h}$. 


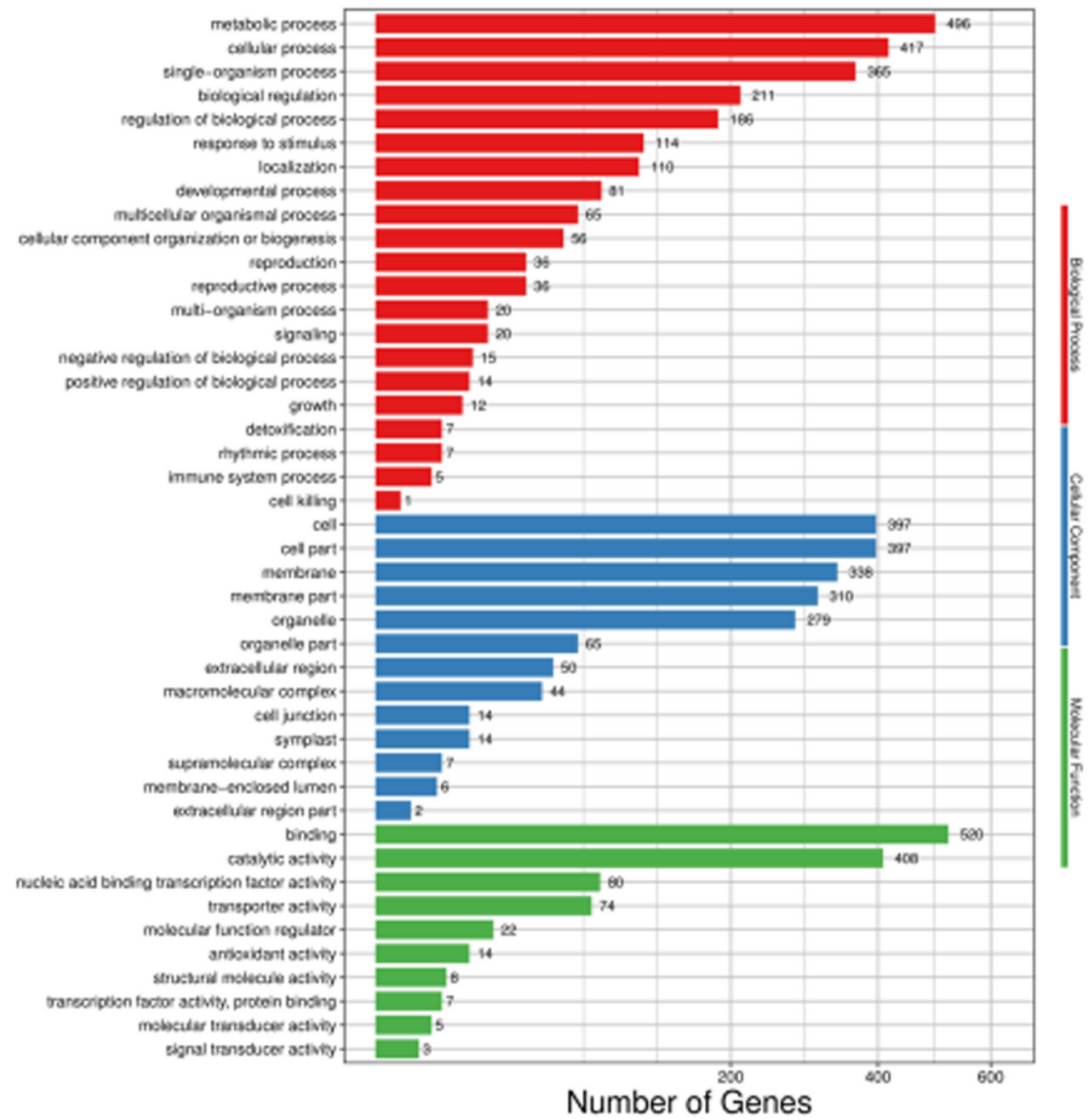

Figure 2

Bar graph of differentially expressed gene GO classification. Red bars represent the biological process, blue bars indicate the cellular component, and green bars represent the molecular function. 


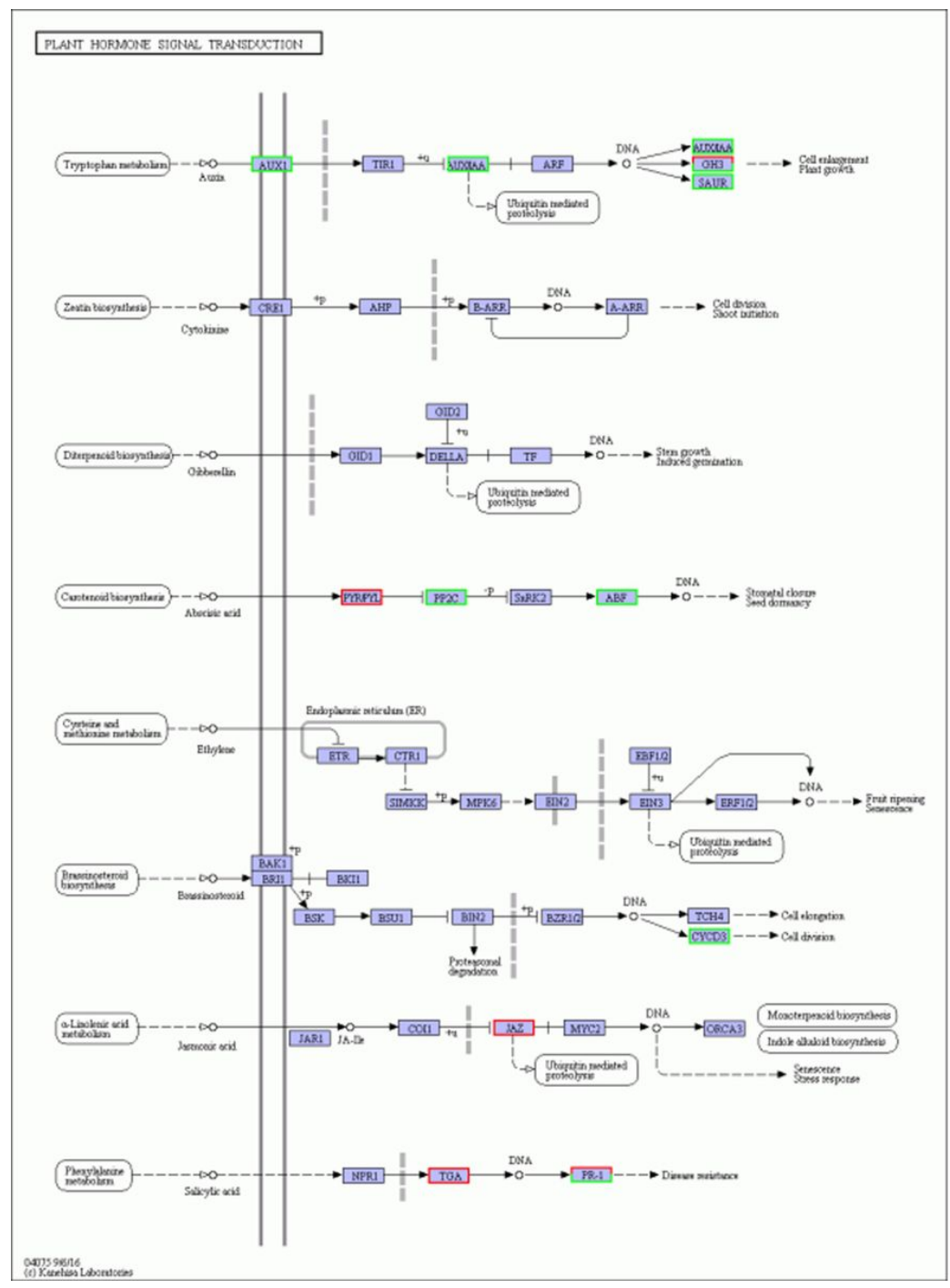

Figure 3

Plant hormone signal transduction pathway inA. chinensis tetraploid plants treated with low-temperature. Red box, up-regulated genes; green box, downregulated genes; blue bx, both. 


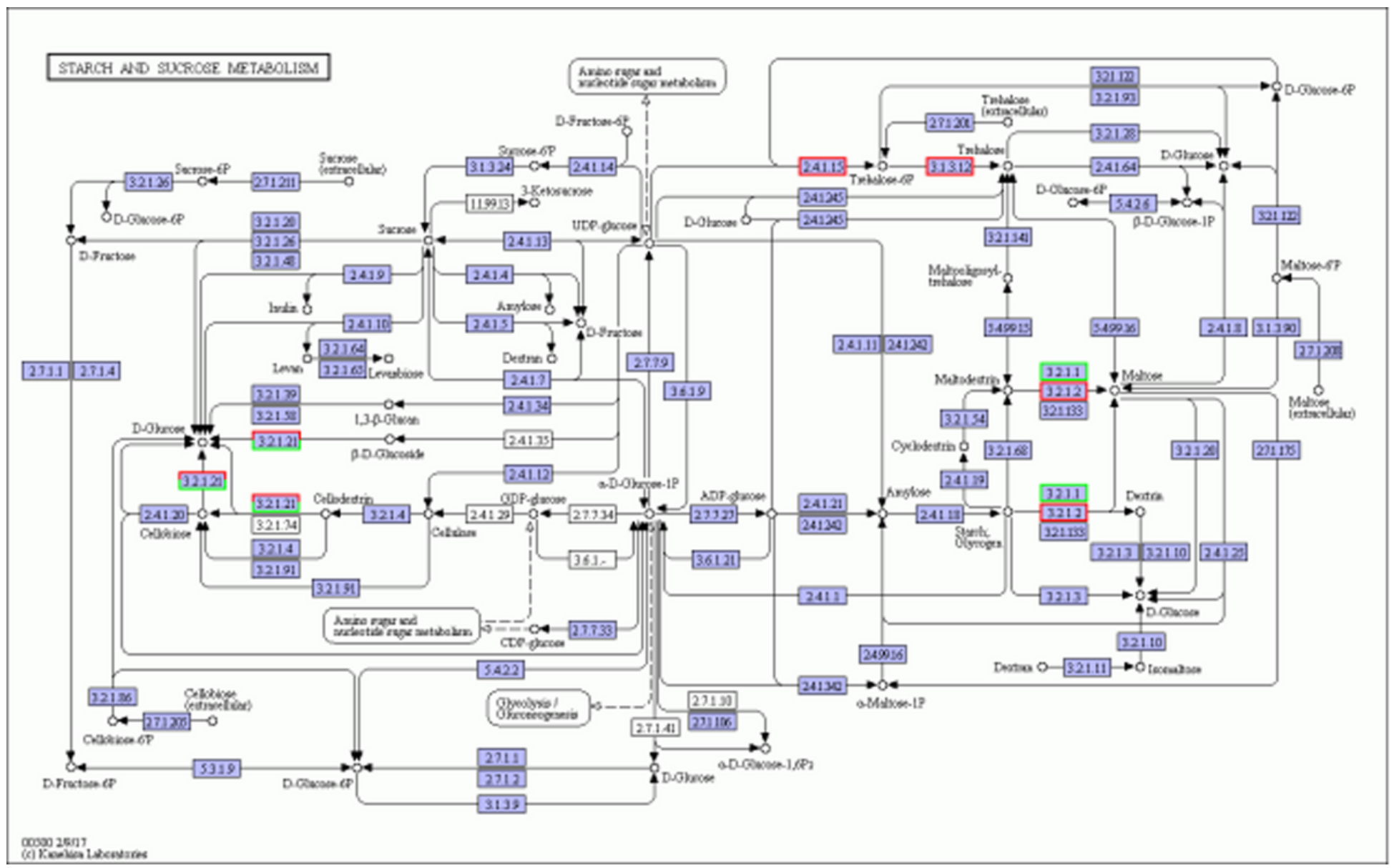

Figure 4

Starch and sucrose metabolic pathwayin A. chinensis tetraploid plants treated with low-temperature. Red box, up-regulated genes; green box, downregulated genes; blue box, both.

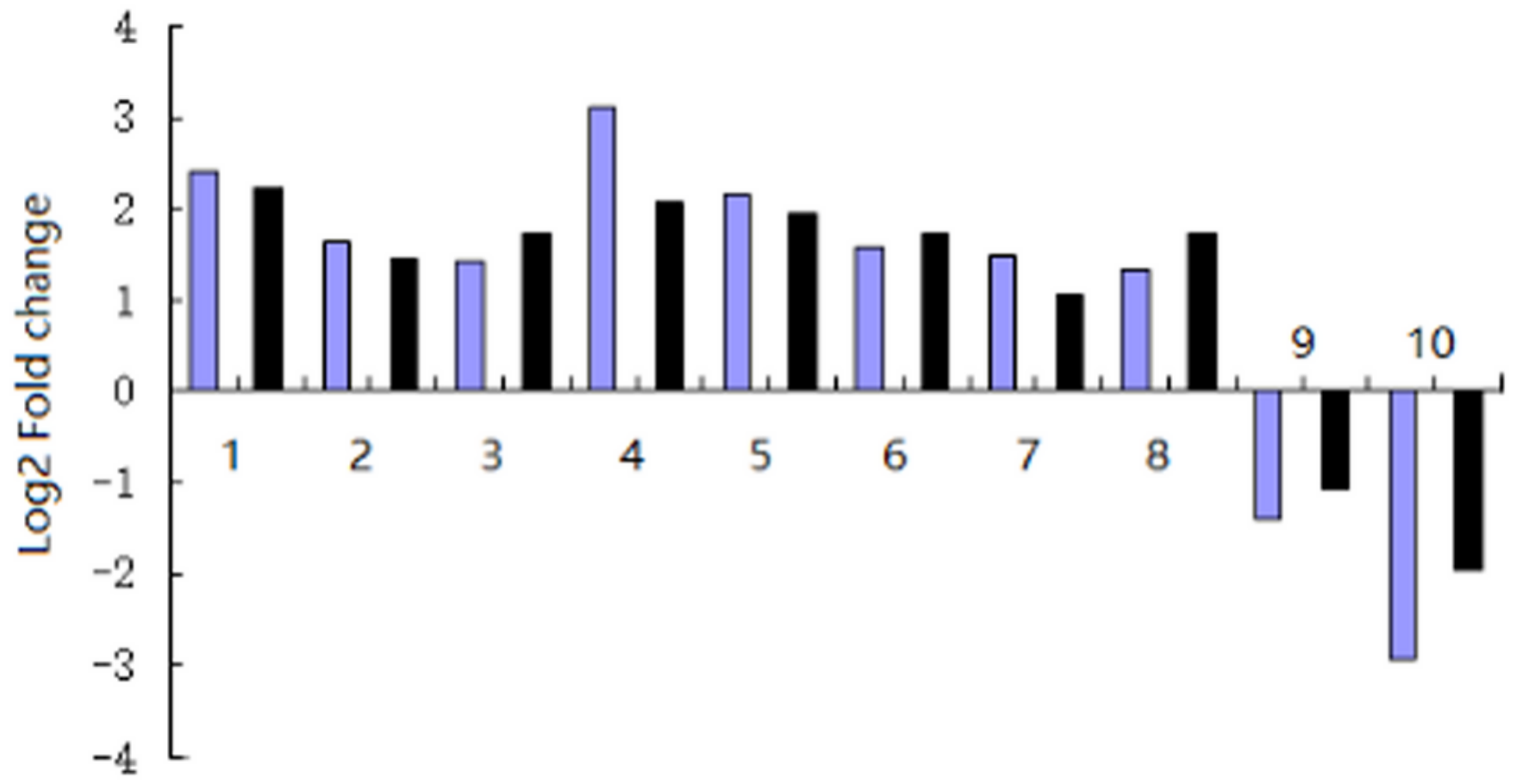

Figure 5 
Comparison of RNA-sequencing and RT-qPCR results of selected DEGs. , RNA-seq; , RT-qPCR. 1, Abscisic acid receptor PYL; 2, BZIP transcription factor family protein; 3, Protein TIFY; 4, Alpha-trehalose-phosphate synthase; 5, Beta-amylase; 6, Beta-glucosidase $1 \mathrm{GH} 1$ family; 7 , Trehalose-phosphate phosphatase F; 8, Beta-amylase 4; 9, Beta-glucosidase 12-like; 10, Alpha-amylase. RT-qPCR was performed on 3 low-temperature treated and 3 control plantlets, normalized with housekeeping gene Elongation factor, repeated 3 times. DEG, differentially expressed gene; RT-qPCR, Real time quantitative polymerase chain reaction. 